\title{
Journal of Sensor and Actuator Networks
}

ISSN 2224-2708

www.mdpi.com/journal/jsan

Article

\section{Sequential Hypothesis Testing Based Approach for Replica Cluster Detection in Wireless Sensor Networks}

\section{Jun-Won Ho}

Department of Information Security, Seoul Women's University, 621 Hwarangro, Nowon-Gu, Seoul, Korea; E-Mail: jwho@swu.ac.kr; Tel.: 82-2-970-5607

Received: 10 July 2012; in revised form: 21 August 2012 / Accepted: 27 August 2012 /

Published: 5 September 2012

\begin{abstract}
In wireless sensor networks, replica node attacks are very dangerous because the attacker can compromise a single node and generate as many replicas of the compromised node as he wants, and then exploit these replicas to disrupt the normal operations of sensor networks. Several schemes have been proposed to detect replica node attacks in sensor networks. Although these schemes are capable of detecting replicas that are widely spread in the network, they will likely fail to detect replica cluster attacks in which replicas form a cluster in a small region. These attacks are also harmful because the attacker can leverage a replica cluster to harmfully impact on the much of the network. To defend against replica cluster attacks, we propose an efficient and effective replica cluster detection scheme using the Sequential Hypothesis Testing. We evaluate our proposed scheme through analysis and simulation. The evaluation results demonstrate that it accomplishes robust replica cluster detection capability.
\end{abstract}

Keywords: replica cluster attacks; sequential hypothesis testing; wireless sensor networks

\section{Introduction}

Wireless sensor networks are very useful for performing a variety of tasks. In particular, they are well suited to monitor and sense natural phenomenon and are useful for projects such as earthquake detection, biohazard detection, flood detection, weather forecasting, and fire detection. In addition to these applications, wireless sensor networks can also be deployed for different type of civilian and military applications such as border line surveillance, intrusion detection, nuclear and chemical attack detection, traffic surveillance and patient monitoring [1,2]. 
However, wireless sensor networks could face with the risk of being compromised by the attacker. More specifically, sensor nodes could be easily captured and compromised by the attacker when wireless sensor networks are deployed in hostile environment. (According to the research result of [3], attacker needs approximately one minute to compromise a sensor node.) The attacker can exploit the compromised nodes to mount various attacks [4,5] against sensor networks. For example, he can disrupt various network operations such as clustering, localization, routing, and time synchronization. He can also inject false data into the network in order to undermine the normal data-acquisition process. Moreover, he can launch jamming attacks to interfere with the communications between sensor nodes.

To generate the widespread effects on the network, he can mount replica node attacks [6] with the compromised nodes. More specifically, he can obtain the secret keying materials and ID from a compromised node. Using these secret keying materials and ID, he can produce replica nodes, which are sensor nodes that share the compromised node's secret keying materials and ID, and then spread these replicas throughout the network and launch various types of attacks by exploiting these replicas. These replica node attacks are very hazardous to the operation of sensor networks because they make it possible for the attacker to gain control over much of the network. Specifically, replicas have the same secret keying materials and IDs as the compromised node and thus they would be treated as the original compromised nodes. This means that they could be allowed to communicate with other nodes and the base station as if they were legitimate nodes. By leveraging this insider position of the replicas, the attacker with a large number of replica nodes could acquire control over much of the network and thus quickly undermine many different types of normal operations in sensor networks.

Several replica node detection schemes [6-11] have been proposed for wireless sensor networks. The primary method used by the majority of these schemes is to have nodes report location claims that identify their positions and attempt to detect colliding reports that indicate one node in multiple locations. Since this method leverages the location information for replica detection, it cannot work without the help of secure localization or GPS techniques. If we could detect replicas without the aid of these techniques, we would save the detection costs subject to employing these techniques.

Indeed, we could detect replicas without the use of location information in case of replica cluster attacks, in which multiple replicas of a single compromised node form a cluster in such a way that they are placed close to each other in the cluster. Specifically, in replica cluster attacks, multiple replicas with the same identity and secret keying materials are placed in the same small regions. All of these replicas want to maximize their malicious impact on the network and thus communicate with as many nodes as possible at the same time. Accordingly, it is highly likely that the number of nodes with which these replicas would communicate at a time would be much more than the one of their benign neighbors. By leveraging this intuition, every node performs the Sequential Probability Ratio Test (SPRT) [12] on its neighbor node using a null hypothesis that a replica cluster of the neighbor node does not exist and an alternate hypothesis that a replica cluster of the neighbor node exists. In using the SPRT, if the number of communication peers of a neighbor node falls short of or exceeds a pre-configured threshold, it will lead to acceptance of the null or alternate hypotheses, respectively. Once the alternate hypothesis is accepted, the node will disconnect the communication with the neighbor node. (A preliminary version of this work appeared in [13].) 
We analytically show that our proposed scheme fulfills high replica cluster detection capability with a few number of samples. The simulation results also demonstrate that it detects replica clusters with at most 3.28 and 6.78 samples while reaching zero false positive and false negative rates.

The rest of paper is organized as follows. In Section 2, we describe the related works. In Section 3, we describe the network assumptions and attacker models that are necessary for our proposed scheme. We also present an overview of the SPRT. In Section 4, we propose a replica cluster detection scheme in sensor networks. In Section 5, we analyze the performance and security of our scheme. In Section 6, we present the simulation results of our proposed scheme. In Section 7, we conclude the paper.

\section{Related Work}

In this section, we present the related work for replica detection in sensor networks. Parno et al. [6] proposed randomized and line-selected multicast schemes to detect replicas in sensor networks. In the randomized multicast scheme, every node is required to multicast a signed location claim to randomly chosen witness nodes. A witness node that receives two colliding location claims for a node decides that the node has been replicated and starts a revocation process on the node. The line-selected multicast scheme diminishes communication overhead of the randomized multicast scheme by controlling every claim-forwarding node to get involved in the replica detection and revocation process. Conti et al. [8] proposed a new method for choosing witness nodes for replica detection. This method improves the replica detection probability, storage and computation overheads of the line-selected multicast scheme in [6]. Zhu et al. [11] proposed localized multicast schemes based on grid cell topology. In this scheme, a location claim is multicast to a single cell or multiple cells and a cell receiving two conflicting locations claims initiates replica detection and revocation process. It achieves higher detection rates and lower storage overheads than the line-selected multicast scheme of [6]. Zeng et al. [10] proposed a random-walk based replica node detection scheme. This scheme uses random walks as the distribution mechanism of location claims in the network. In this scheme, location claims are highly randomly distributed to witness nodes.

Choi et al. [7] proposed a replica detection scheme in which the network is divided into a set of non-overlapping subregions and an exclusive subset is formed in each subregion. If the intersection of subsets is not empty, it signals that replicas are contained in those subsets.

Xing et al. [9] proposed a fingerprint-based replica node detection scheme. In this scheme, nodes inform the base station of fingerprints, which identify a set of their neighbors. The base station performs replica detection by using the property that fingerprints of replicas collide with each other.

Ho et al. [14] leverages group deployment knowledge to detect static replica nodes with little overhead.

Ho et al. [15] adapts the SPRT to tackle a replica node detection problem in mobile sensor networks.

Yu et al. [16]proposed a mobile replica detection scheme by leveraging the intuition that the number of mobile nodes contacted by mobile replicas in a time slot is larger than the one contacted by a benign mobile node.

Wang et al. [17] detects the replicas nodes by using mobile patrollers in sensor networks.

Bonaci et al. [18] proposed an optimization framework for replica node detection in sensor networks. 
Although the related work could effectively detect replica nodes that are widely spread over the network, they likely treat the replicas in a cluster as a single node and thus would likely fail to detect replica clusters. To justify this argument, we first assume that multiple replicas form a cluster $C$ such that they are placed in a circle whose center is $(x, y)$ and radius is $r$. Moreover, we assume that there are multiple neighbor nodes outside the circle cluster $C$ and the longest distance from these neighbor nodes to the circumference of cluster $C$ is $l$. Let us denote $R$ be a communication radius of a sensor node. As long as $2 r+l \leq R$ holds, each neighbor node outside the cluster $C$ can directly communicate each replica in the cluster $C$. Putting it in differently, if all replicas in the cluster $C$ claim the same location $(x, y)$ irrespective of their actual locations, they could cheat the neighbor nodes outside the cluster $C$ as if they were a single node placed in $(x, y)$. Hence, this will result in the failure of replica cluster detection. Our proposed scheme mitigates this limitation of the related work by efficiently adapting the SPRT to the replica cluster detection problem.

\section{Preliminaries}

In this section, we first present the network assumptions and then describe our attacker models. Finally, we present an overview of the SPRT.

\subsection{Network Assumptions}

We assume a static sensor network where each sensor node is fixed to its initial deployment position. Although sensor nodes are static, their neighboring nodes could be changed because the network operator could replace the dead nodes with new ones or keep performing additional node deployments for the better sensing operations. Moreover, every sensor node is assumed to be capable of identifying the destinations of all messages originating from its neighbors. This can be achieved by letting node operate in promiscuous mode. More specifically, every node collects only the source and destination node information of each incoming packet while not looking into the main contents of the packet.

\subsection{Attacker Models}

We assume that an adversary can compromise a subset of sensor nodes and have full control of them. In other words, an adversary can leverage the compromised nodes to perform any malicious activities that damage the network. Moreover, he can create the widespread effects on the network by generating many replicas of the compromised nodes, where a replica node is defined as a node having the same ID and secret keying materials as a compromised node. In terms of replica node deployment, we assume that adversary will attempt to form a cluster consisting of multiple replicas of a single compromised node in a small region, and then launch a variety of types of attacks by leveraging replica cluster. To amplify the impacted regions by replica cluster attacks, he can compromise multiple nodes, create multiple replica clusters in such a way that each replica cluster is constructed from each compromised node, and place multiple replica clusters in the different small regions. 


\subsection{Sequential Probability Ratio Test (SPRT)}

The Sequential Probability Ratio Test (SPRT) [12] is a statistical decision process that comes to a decision with multiple pieces of evidence. It is also considered to be one-dimensional random walk with lower and upper limits [19]. Specifically, random walk starts from a point between two limits and moves toward the lower or upper limit in line with the type of each incoming evidence. If the walk comes to (or exceeds) the lower (resp. upper) limit, it terminates and accepts the null (resp. alternate) hypothesis.

The main benefit of using the SPRT is that it requires only few pieces of evidence to make a correct decision at the cost of low false positive rate and false negative rate [12].

\section{Replica Cluster Attack Detection}

In replica cluster attacks, multiple replicas with the same ID and secret keying materials are closely deployed to each other in a small region. Since the replicas in a cluster want to affect maliciously as many nodes as possible in the network, they would actively communicate with as many nodes as possible at the same time. Hence, the replicas in a cluster would likely communicate with more peers at a time than the ones of their benign neighbors. By leveraging this intuition, we adapt the SPRT to tackle the replica cluster detection problem. Specifically, every node performs the SPRT on its neighbor node with a null hypothesis that a replica cluster of the neighbor node does not exist and an alternate hypothesis that a replica cluster of the neighbor node exists. In the SPRT, as the number of communication peers of a neighbor node falls short of (resp. exceeds) a pre-configured threshold, the random walk will move toward the lower (resp. upper) limit, leading to the acceptance of the null (resp. alternate) hypothesis. Once the SPRT accepts the alternate hypothesis, the node will stop the communication with the neighbor node. The replica cluster detection procedure is described as follows.

After deployment, every sensor node $u$ discovers its neighboring nodes. Node $u$ monitors all messages originating from every neighbor node $v$ and identifies the destinations of these messages. Assume that the entire time domain of $u$ is divided into a series of time slots. Node $u$ computes $X_{i}(i \geq 1)$, which is defined as the number of distinct destinations of all messages from $v$ during the $i$ th time slot.

Let $N$ be a total number of nodes in the network. Let $R_{i}$ be a Bernoulli random variable that is defined as:

$$
R_{i}= \begin{cases}0 & \text { if } \frac{X_{i}}{N}<\rho \\ 1 & \text { if } \frac{X_{i}}{N} \geq \rho\end{cases}
$$

The success probability $p$ of Bernoulli distribution is defined as:

$$
p=\operatorname{Pr}\left(R_{i}=1\right)=1-\operatorname{Pr}\left(R_{i}=0\right)
$$

If $p$ is smaller than or equal to a preset threshold $p_{0}$, it is likely that a replica cluster of $v$ does not exist. On the other hand, if $p$ is greater than or equal to a preset threshold $p_{1}\left(p_{0}<p_{1}\right)$, it is likely that a replica cluster of $v$ exists. The problem of deciding whether a replica cluster of $v$ exists can be formulated as a hypothesis testing problem with null and alternate hypotheses of $p \leq p_{0}$ and $p \geq p_{1}$, respectively. In this problem, we define a false positive $\alpha$ (resp. a false negative $\beta$ ) as the likelihood that the alternate (resp. null) hypothesis is erroneously accepted when $p \leq p_{0}$ (resp. $p \geq p_{1}$ ). In order to make a correct decision 
in hypothesis testing problem, the false positive (resp. false negative) does not exceed user-configured false positive (resp. user-configured false negative).

In line with this sampling plan, we present how a node $u$ performs the SPRT to decide about a node $v$ from the $n$ observed samples, which are $R_{1}, R_{2}, \ldots, R_{n}$. We first define the null hypothesis $H_{0}$ and the alternate one $H_{1}$ as follows: $H_{0}$ is the hypothesis that a replica cluster of a node $v$ does not exist and $H_{1}$ is the hypothesis that a replica cluster of a node $v$ exists. We then define $L_{n}$ as the log-probability ratio on $n$ samples, given as:

$$
L_{n}=\ln \frac{\operatorname{Pr}\left(R_{1}, \ldots, R_{n} \mid H_{1}\right)}{\operatorname{Pr}\left(R_{1}, \ldots, R_{n} \mid H_{0}\right)}
$$

Assume that $R_{i}$ is independent and identically distributed. Then $L_{n}$ can be rewritten as:

$$
L_{n}=\ln \frac{\prod_{i=1}^{n} \operatorname{Pr}\left(R_{i} \mid H_{1}\right)}{\prod_{i=1}^{n} \operatorname{Pr}\left(R_{i} \mid H_{0}\right)}=\sum_{i=1}^{n} \ln \frac{\operatorname{Pr}\left(R_{i} \mid H_{1}\right)}{\operatorname{Pr}\left(R_{i} \mid H_{0}\right)}
$$

Let $\theta_{n}$ denote the number of times that $R_{i}=1$ in the $n$ samples. Then we have

$$
L_{n}=\theta_{n} \ln \frac{p_{1}}{p_{0}}+\left(n-\theta_{n}\right) \ln \frac{1-p_{1}}{1-p_{0}}
$$

where

$$
p_{0}=\operatorname{Pr}\left(R_{i}=1 \mid H_{0}\right), \quad p_{1}=\operatorname{Pr}\left(R_{i}=1 \mid H_{1}\right), \quad p_{0}<p_{1}
$$

Based on the log-probability ratio $L_{n}$, the SPRT for $H_{0}$ against $H_{1}$ is given as follows:

- $L_{n} \leq \ln \frac{\beta^{\prime}}{1-\alpha^{\prime}}:$ accept $H_{0}$ and terminate the test;

- $L_{n} \geq \ln \frac{1-\beta^{\prime}}{\alpha^{\prime}}:$ accept $H_{1}$ and terminate the test;

- $\ln \frac{\beta^{\prime}}{1-\alpha^{\prime}}<L_{n}<\ln \frac{1-\beta^{\prime}}{\alpha^{\prime}}$ : continue the test process with another observation,

We can rewrite the SPRT as follows:

- $\theta_{n} \leq \tau_{0}(n)$ : accept $H_{0}$ and terminate the test;

- $\theta_{n} \geq \tau_{1}(n)$ : accept $H_{1}$ and terminate the test;

- $\tau_{0}(n)<\theta_{n}<\tau_{1}(n)$ : continue the test process with another observation,

where

$$
\tau_{0}(n)=\frac{\ln \frac{\beta^{\prime}}{1-\alpha^{\prime}}+n \ln \frac{1-p_{0}}{1-p_{1}}}{\ln \frac{p_{1}}{p_{0}}-\ln \frac{1-p_{1}}{1-p_{0}}}, \tau_{1}(n)=\frac{\ln \frac{1-\beta^{\prime}}{\alpha^{\prime}}+n \ln \frac{1-p_{0}}{1-p_{1}}}{\ln \frac{p_{1}}{p_{0}}-\ln \frac{1-p_{1}}{1-p_{0}}}
$$

If the SPRT decides that a replica cluster of $v$ does not exist, $u$ restarts the SPRT with newly measured samples from $v$. However, if the SPRT decides that a replica cluster of $v$ exists, $u$ terminates the SPRT on $v$ and isolates $v$ from the network by stopping communication with $v$.

\section{Analysis}

In this section, we first describe the replica cluster detection accuracy of our proposed scheme and then analyze the resilience of our proposed scheme against replica cluster attacks. Next, we show how many observations on average are required to detect replica clusters. Finally, we provide an analysis on the energy consumption of our scheme. 


\subsection{Replica Cluster Detection Accuracy}

In the SPRT, we define two types of errors as follows:

- $\alpha$ : false positive probability that the SPRT leads to the acceptance of $H_{1}$ when $H_{0}$ is true.

- $\beta$ : false negative probability that the SPRT leads to the acceptance of $H_{0}$ when $H_{1}$ is true.

Since $\beta$ is the false negative probability, $(1-\beta)$ is the replica cluster detection probability. According to [12], the following inequality holds:

$$
(1-\beta) \geq \frac{1-\alpha^{\prime}-\beta^{\prime}}{1-\alpha^{\prime}}
$$

where $\alpha^{\prime}$ and $\beta^{\prime}$ are user-configured false positive and false negative probabilities. Thus, replica cluster detection probability is bounded from below by $\frac{1-\alpha^{\prime}-\beta^{\prime}}{1-\alpha^{\prime}}$.

From Equation (5), we see that low user-configured false positive and false negative probabilities will result in a low false negative probability for the sequential test process, leading to high detection rates.

\subsection{Resilience against Replica Cluster Attacks}

Let us investigate how our proposed scheme tolerates against replica cluster attacks.

Lemma 5.1 Let $\gamma$ denote the faction of the samples with $H_{1}$ type out of $n$ samples that node $u$ receives from a replica cluster of node $v$. Given $n$ samples, $u$ detects a replica cluster of node $v$ if $u$ receives at least $\gamma^{*} \times n$ samples with $H_{1}$ type from a replica cluster of $v$ such that $\gamma^{*}=\frac{\ln \frac{1-\beta^{\prime}}{\alpha^{\prime}}+n \ln \frac{1-p_{0}}{1-p_{1}}}{\left(\ln \frac{p_{1}}{p_{0}}-\ln \frac{1-p_{1}}{1-p_{0}}\right) n}$.

Proof 5.1 Since $\theta_{n}$ is the number of times that the sample with $H_{1}$ type occurs in $n$ samples, $\theta_{n}=\gamma \times n$ holds. According to the SPRT, u detects a replica cluster of $v$ if $\theta_{n} \geq \tau_{1}(n)$, which is rewritten as $\gamma \geq \gamma^{*}$ such that $\gamma^{*}=\frac{\ln \frac{1-\beta^{\prime}}{\alpha^{\prime}}+n \ln \frac{1-p_{0}}{1-p_{1}}}{\left(\ln \frac{p_{1}}{p_{0}}-\ln \frac{1-p_{1}}{1-p_{0}}\right) n}$. Hence, if the number of samples with $H_{1}$ type that $u$ receives from $a$ replica cluster of $v$ is at least $\gamma^{*} \times n$, u detects a replica cluster of $v$.

Let us explore how $n$ affects $\gamma^{*}$ when $\alpha^{\prime}=\beta^{\prime}=0.01, p_{0}=0.1$, and $p_{1}=0.9$. As shown in Figure 1, we see that $\gamma^{*}$ gradually decreases as $n$ increases. This indicates that the SPRT requires the lower fraction of samples with $H_{1}$ types for replica cluster detection as the total number of samples increases. Putting it differently, the SPRT detects replica cluster attacks with the fewer number of samples as a replica cluster more actively makes a malicious impact on the network.

\subsection{Average Number of Samples for Decision}

Let $n$ denote the number of samples to terminate the SPRT. Since $n$ is changed with the types of samples, it is considered as a random variable with an expected value $E[n]$. According to [12], we obtain $E[n]$ as follows.

$$
E[n]=\frac{E\left[L_{n}\right]}{E\left[\ln \frac{\operatorname{Pr}\left(R_{i}^{j} \mid H_{1}\right)}{\operatorname{Pr}\left(R_{i}^{j} \mid H_{0}\right)}\right]}
$$


From this equation, we compute the expected numbers of $n$ conditioned on the hypotheses $H_{0}$ and $H_{1}$ as follows:

$$
\begin{aligned}
& E\left[n \mid H_{0}\right]=\frac{\left(1-\alpha^{\prime}\right) \ln \frac{\beta^{\prime}}{1-\alpha^{\prime}}+\alpha^{\prime} \ln \frac{1-\beta^{\prime}}{\alpha^{\prime}}}{p_{0} \ln \frac{p_{1}}{p_{0}}+\left(1-p_{0}\right) \ln \frac{1-p_{1}}{1-p_{0}}} \\
& E\left[n \mid H_{1}\right]=\frac{\beta^{\prime} \ln \frac{\beta^{\prime}}{1-\alpha^{\prime}}+\left(1-\beta^{\prime}\right) \ln \frac{1-\beta^{\prime}}{\alpha^{\prime}}}{p_{1} \ln \frac{p_{1}}{p_{0}}+\left(1-p_{1}\right) \ln \frac{1-p_{1}}{1-p_{0}}}
\end{aligned}
$$

Note that a sample in the SPRT corresponds to a time slot and thus $E\left[n \mid H_{0}\right]$ and $E\left[n \mid H_{1}\right]$ represent an average number of time slots to terminate the SPRT conditioned on the hypothesis $H_{0}$ and $H_{1}$, respectively. From Equation (7), given a value of $p_{1}, E\left[n \mid H_{1}\right]$ increases as $p_{0}$ rises. This means that $p_{0}$ needs to be configured as a small value in order for replica clusters to be detected with a small number of samples. On the other hand, given a value of $p_{0}, E\left[n \mid H_{1}\right]$ decreases as $p_{1}$ increases. This implies that the large value of $p_{1}$ diminishes the number of claims required for replica cluster detection.

Figure 1. The effects of $n$ on $\gamma^{*}$, which is the minimum fraction of samples with type $H_{1}$ in $n$ samples required for node $u$ to detect a replica cluster of node $v$.

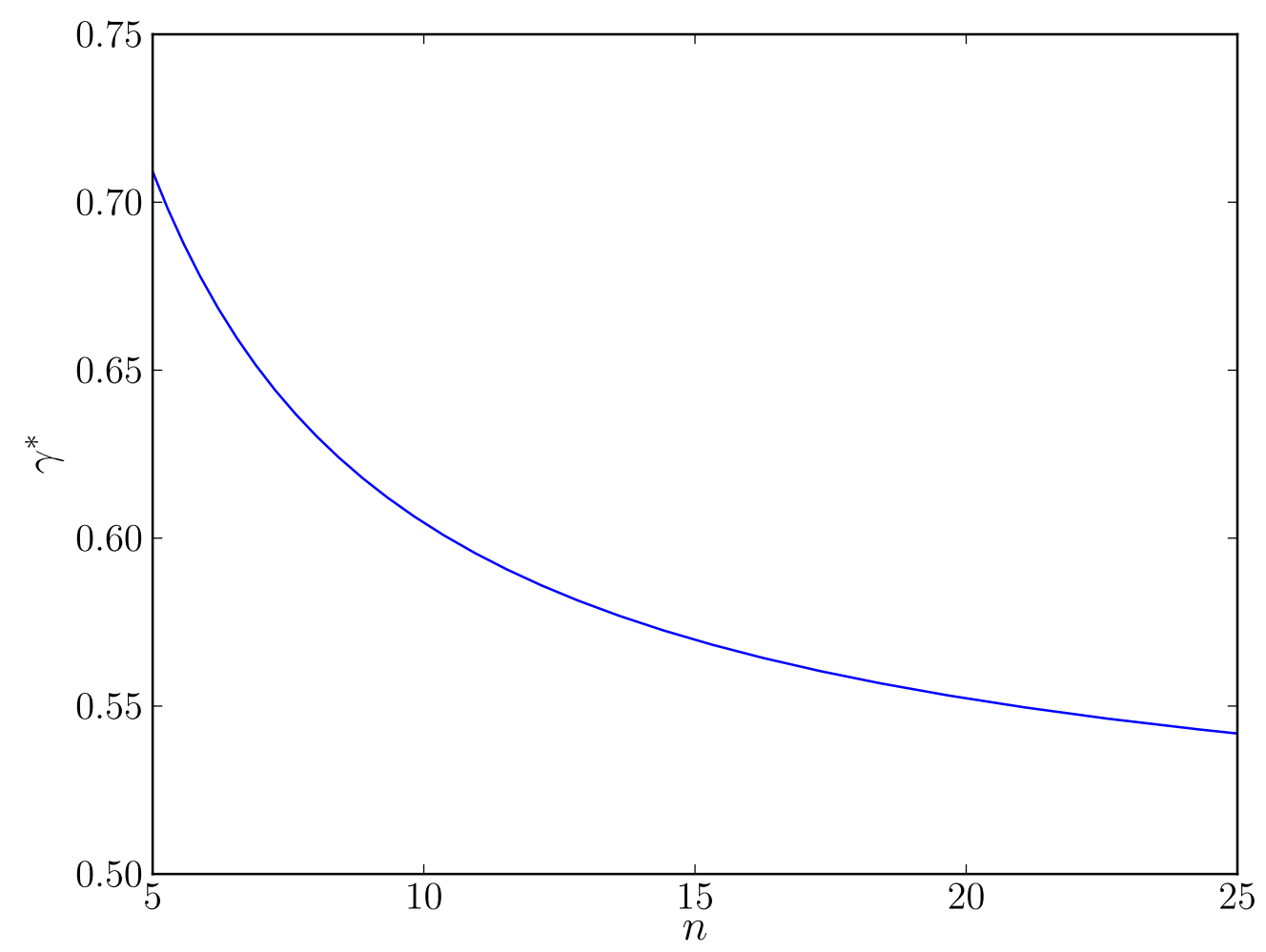

\subsection{Energy Consumption}

Since our scheme requires each sensor node to operate in monitor mode, each sensor node needs to receive all packets sent by its neighboring nodes. We compute the amount of energy that a senor node consumes to receive the packets. According to [20], Mica2 sensor mote consumes $10 \mathrm{~mA}$ current to receive a byte. When battery voltage is $3 \mathrm{~V}$ and data rate is $19.2 \mathrm{Kbps}$, Mica2 takes $\frac{1}{2.4 \times 10^{3}}$ seconds to receive a byte. Thus, it consumes $0.03 / 2.4 \times 10^{3} \mathrm{~J}=12.5 \mu \mathrm{J}$ to receive a byte. Based on this result, 
we compute the amount of energy depleted by a node when it receives the packets from its neighbors. For this calculation, we consider three cases of the packet size such as 12, 24, 36 bytes. As shown in Figure 2, as the number of packets received by a node increases, the amount of energy consumed by a node also linearly increases. Given a number of received packets, we observe that the amount of energy consumption rises in line with an increase of packet size. From this observation, we see that packet size needs to be configured to be a small value in order to reduce energy consumption.

Figure 2. Energy consumption $v s$. number of packets received by a node.

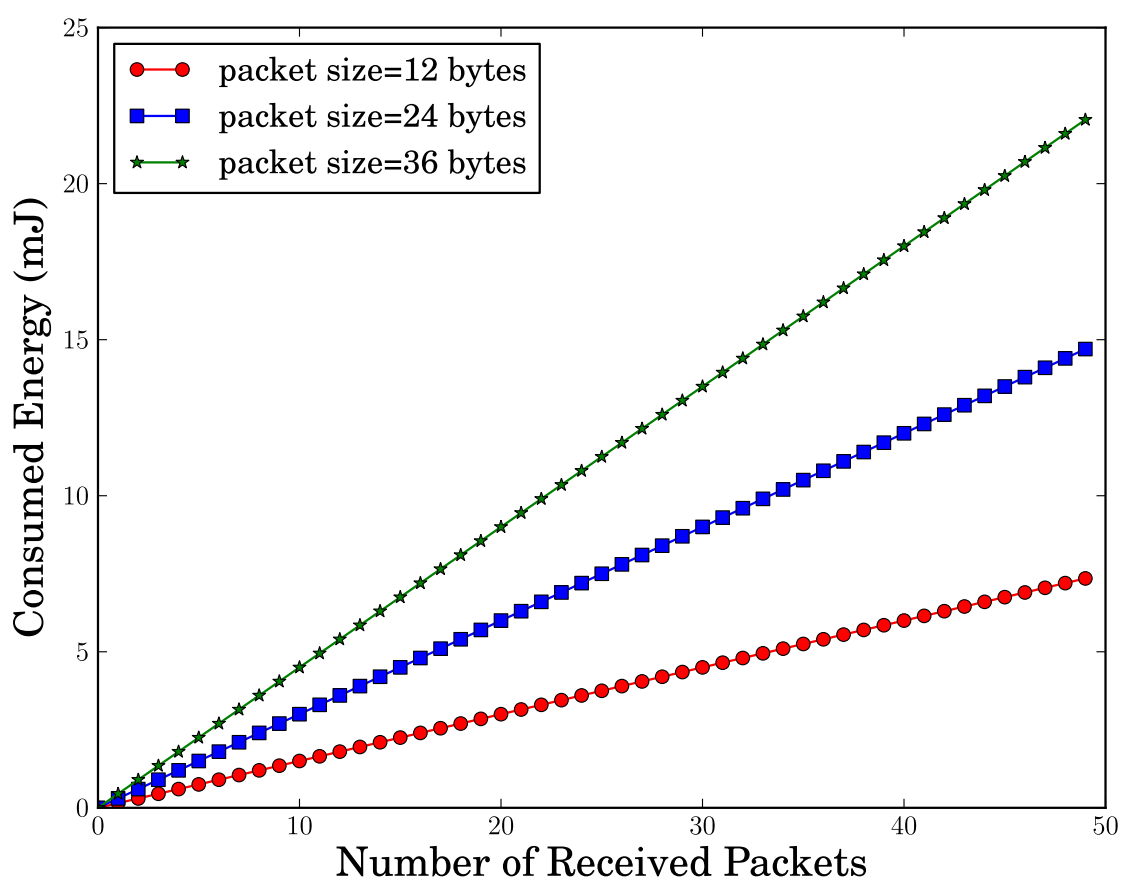

\section{Simulation Study}

In this section, we first describe our simulation environment and then present the simulation results.

\subsection{Simulation Environment}

We developed a simple simulation program to evaluate our proposed scheme. In our simulation, we have 1,000 sensor nodes and set $\rho=0.01$. We divide the entire time domain into 60 time slots such that a time slot duration is 60 simulation seconds and perform the simulation in units of time slots. We evaluate our scheme in two cases: benign and replica cluster. In benign case, a benign source node communicates with a number of destination nodes. In replica cluster case, a set of replica nodes with the same ID and secret keying materials forms a cluster, which communicates with a number of destination nodes. In both cases, we model the occurrence of the communication with a distinct destination node as a homogeneous Poisson process with rate parameter $\lambda$, and thus the inter-occurrence times of distinct communications follow the exponential distribution. More specifically, the inter-occurrence time between two consecutive communications is calculated as $-\frac{\ln (U)}{\lambda}$, where $U$ is a uniform random variate such that $0 \leq U<1$. In 
benign case, $\lambda$ ranges from 0.02 to 0.1 . In replica cluster case, $\lambda$ ranges from 0.2 to 0.4 . We also set both the user-configured false positive threshold $\alpha$ and the false negative threshold $\beta$ to 0.01 , and we set the lower threshold $p_{0}$ and the upper threshold $p_{1}$ to 0.1 and 0.9 , respectively. The rationale behind these configurations is discussed in Section 5.

\subsection{Simulation Results}

We use the following metrics to evaluate the performance of our proposed scheme:

- Average Number of Samples is the average number of samples required for the SPRT to reach a correct decision.

- False Positive is the error probability that benign node is misidentified as a replica cluster.

- False Negative is the error probability that replica cluster is misidentified as a benign node.

We present the average results for 1,000 runs of the simulation in each configuration such that each run is executed for 60 time slots. For each run, we obtain each metric as the average of the results of the SPRTs that are performed. Note that the SPRT will restart (resp. terminate) if it accepts the null (resp. alternate) hypothesis $H_{0}$ (resp. $H_{1}$ ). Now we present our main findings.

First, we find that there are no false positives and no false negatives. Hence, a replica cluster is always detected and no benign node is misidentified as a replica cluster. This indicates that the SPRT comes to a decision with very high accuracy.

Second, we present the results of the average number of samples. For this purpose, we define true negative as the case that a benign node is correctly determined to be a benign node. We also define true positive as the case that a replica cluster is correctly determined to be a replica cluster. In the true negative case, as shown in Figure 3, the average number of samples reaches its maximum of 3.279 for all configurations of $\lambda$. This means that our proposed scheme requires less than four samples with an average in order to make a correct decision on a benign node. We also observe that a rise in $\lambda$ results in a very slight increase in the average number of samples. This indicates that the average number of samples increases very slightly in proportion to the number of distinct destination nodes.

In the true positive case, as shown in Figure 4, the average number of samples reaches its maximum of 6.782 for all configurations of $\lambda$. This means that our proposed scheme achieves fast replica cluster detection with at most seven samples on average. Since a sample in the SPRT corresponds to a time slot and a time slot duration is 60 simulation seconds, the detection time will be at most 420 simulation seconds. Moreover, we observe that the average number of samples decreases as $\lambda$ increases. We infer from this observation that the higher values of $\lambda$ expedite the decision process to terminate in the acceptance of the alternate hypothesis $H_{1}$.

Finally, Figure 5 shows the probability distribution of the number of samples in case of true positive. In this distribution, we explore the cases of $\lambda=0.2$ and $\lambda=0.25$. In case of $\lambda=0.2$, we observe that in $69.4 \%$ of cases, the number of samples is at most seven. In case of $\lambda=0.25$, we observe that in $82.8 \%$ of cases, the number of samples is at most five. This means that the number of samples in most cases is below or very close to the average. As a result, our proposed scheme detects replica clusters with few samples in most cases. 
Figure 3. Average number of samples vs. $\lambda$ in true negative case.

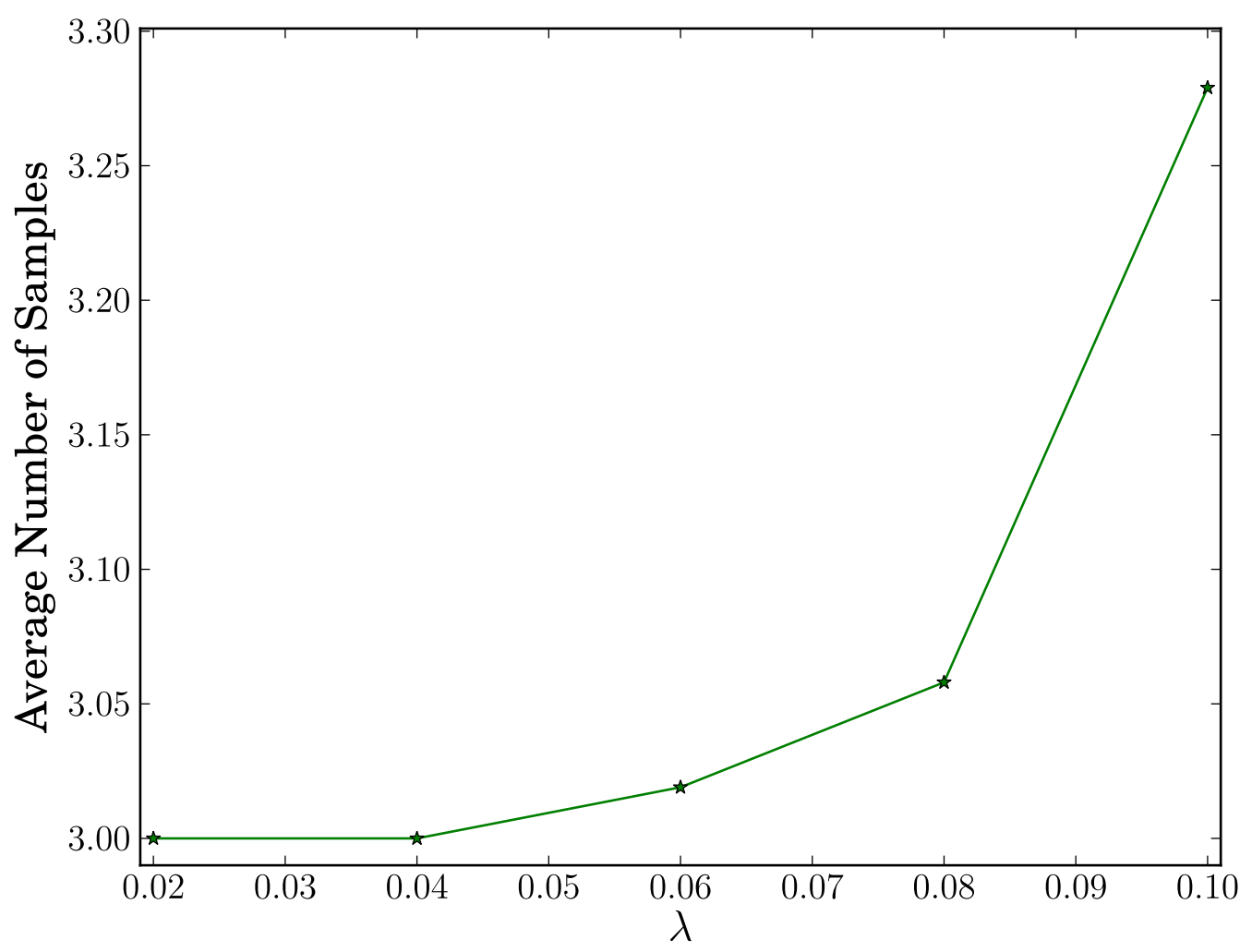

Figure 4. Average number of samples vs. $\lambda$ in true positive case.

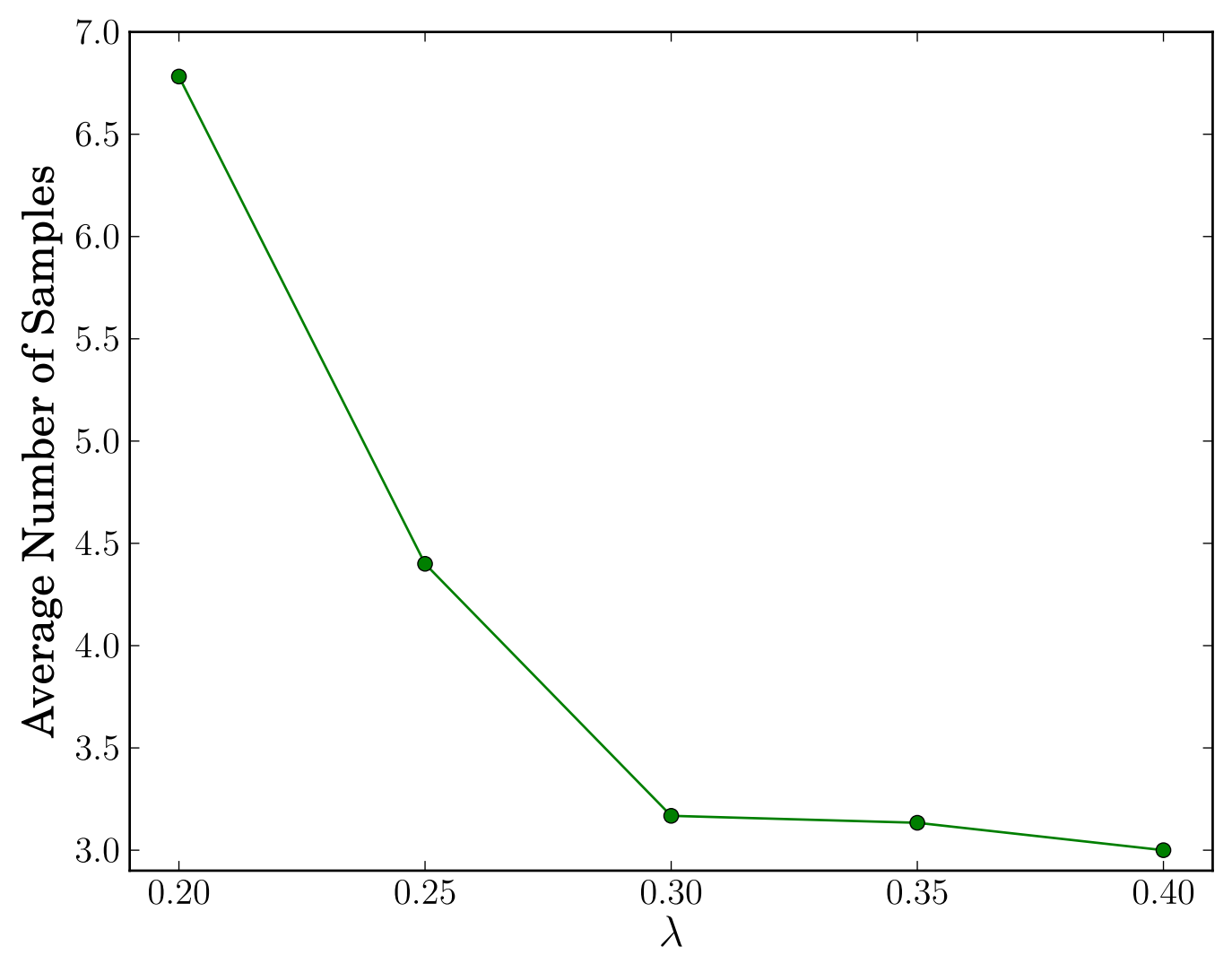


Figure 5. Probability distribution of the number of samples in true positive case.

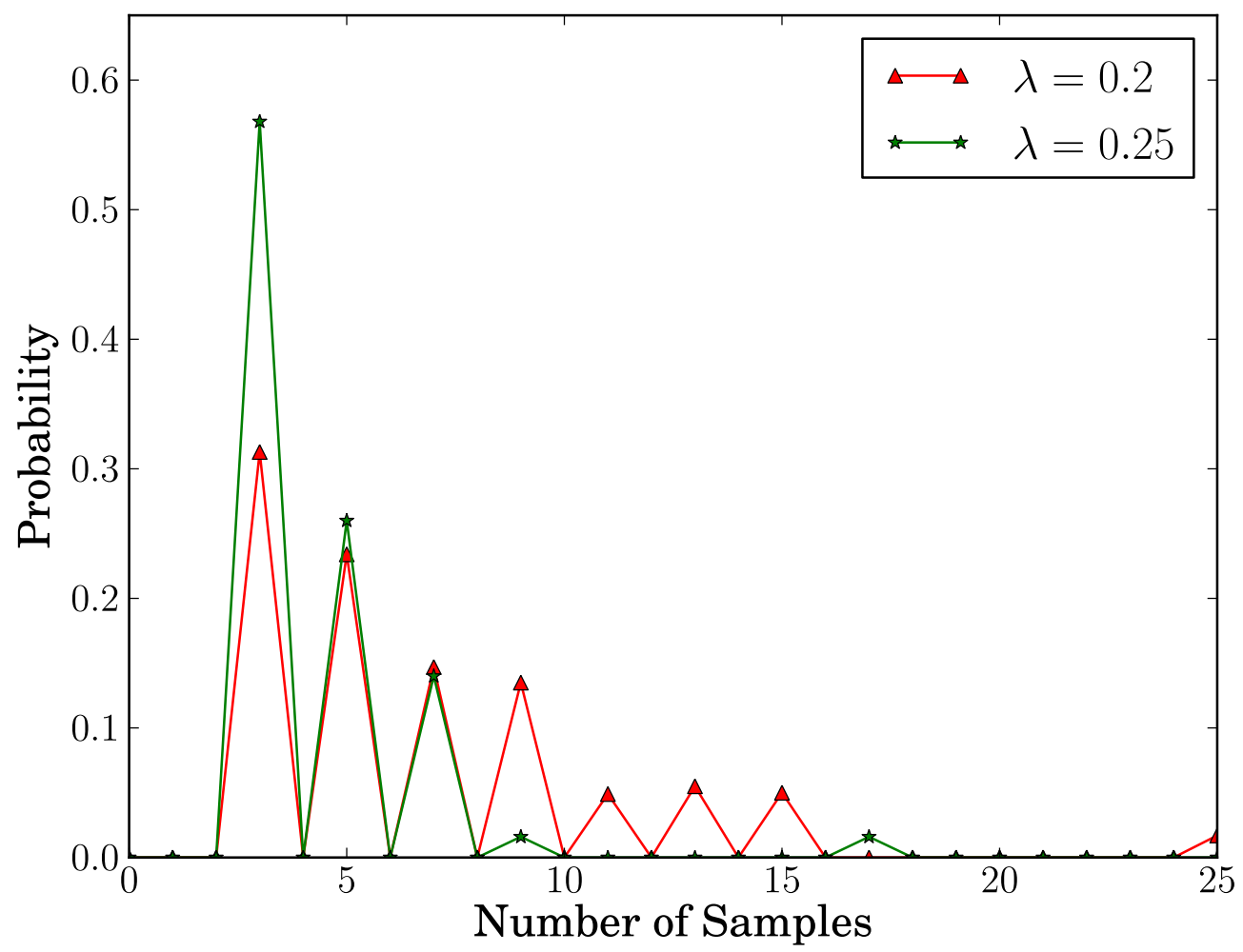

\section{Conclusions}

In this paper, we introduce the replica cluster attacks and propose a replica cluster detection scheme using the Sequential Probability Ratio Test (SPRT). We also analytically show that our proposed scheme achieves robust replica cluster detection capability. Finally, we evaluate our proposed scheme through the simulation. The evaluation results show that it quickly stops replica cluster attacks without false negative or false positive errors.

\section{Acknowledgments}

This work was supported by a research grant from Seoul Women's University (2012).

\section{References}

1. Akyildiz, I.F.; Su, W.; Sankarasubramaniam, Y.; Cayirci, E. Wireless sensor networks: A survey. Comput. Netw. 2002, 38, 393-422.

2. Yick, J.; Mukherjee, B.; Ghosal, D. Wireless sensor network survey. Comput. Netw. 2008, 52, 2292-2330.

3. Hartung, C.; Balasalle, J.; Han, R. Node Compromise in Sensor Networks: The Need for Secure Systems. In Proceedings of the Technical Report CU-CS-990-05, Department of Computer Science, University of Colorado at Boulder, Boulder, CO, USA, January 2005.

4. Karlof, C.; Wagner, D. Secure routing in wireless sensor networks: Attacks and countermeasures. Ad Hoc Netw. J. 2003, 1, 293-315. 
5. Wood, A.D.; Stankovic, J.A. Denial of service in sensor networks. IEEE Comput. 2002, 35, 54-62.

6. Parno, B.; Perrig, A.; Gligor, V.D. Distributed Detection of Node Replication Attacks in Sensor Networks. In Proceedings of the IEEE Symposium on Security and Privacy, Berkeley/Oakland, CA, USA, 8-11 May 2005.

7. Choi, H.; Zhu, S.; La Porta, T.F. SET: Detecting Node Clones in Sensor Networks. In Proceedings of the IEEE/CreateNet Conference on Security and Privacy for Emerging Areas in Communication Networks (SecureComm), Nice, France, 17-21 September 2007.

8. Conti, M.; Pietro, R.D.; Mancini, L.V.; Mei, A. A Randomized, Efficient, and Distributed Protocol for the Detection of Node Replication Attacks in Wireless Sensor Networks. In Proceedings of the 8th ACM International Symposium on Mobile Ad Hoc Networking and Computing, Montreal, Canada, 9-14 September 2007.

9. Xing, K.; Liu, F.; Cheng, X.; Du, H.C. Real-Time Detection of Clone Attacks in Wireless Sensor Networks. In Proceedings of the IEEE ICDCS, Beijing, China, 17-20 June 2008; pp. 3-10.

10. Zeng, Y.; Cao, J.; Zhang, S.; Guo, S.; Xie, L. Random-walk based approach to detect clone attacks in wireless sensor networks. IEEE J. Sel. Areas Commun. 2010, 28, 677-691.

11. Zhu, B.; Addada, V.G.K.; Setia, S.; Jajodia, S.; Roy, S. Efficient Distributed Detection of Node Replication Attacks in Sensor Networks. In Proceedings of the Twenty-Third Annual Computer Security Applications Conference, ACSAC 2007, Miami Beach, FL, USA, 10-14 December 2007.

12. Wald, A. Sequential Analysis; Dover: Mineola, NY, USA, 2004.

13. Ho, J.W. Distributed Detection of Replica Cluster Attacks in Sensor Networks Using Sequential Analysis. In Proceedings of the IEEE International Workshop on Information and Data Assurance (WIDA) in Conjunction with IEEE IPCCC, Austin, TX, USA, 7-9 December 2008.

14. Ho, J.W.; Liu, D.; Wright, M.; Das, S.K. Distributed detection of replica node attacks with group deployment knowledge in wireless sensor networks. Ad Hoc Netw. 2009, 7, 1476-1488.

15. Ho, J.W.; Wright, M.; Das, S.K. Fast detection of mobile replica node attacks in wireless sensor networks using sequential hypothesis testing. IEEE Trans. Mobile Comput. 2011, 10, 767-782.

16. Yu, C.M.; Lu, C.S.; Kuo, S.Y. Efficient and Distributed Detection of Node Replication Attacks in Mobile Sensor Networks. In Proceedings of the IEEE Vehicular Technology Conference Fall (VTC 2009-Fall), Anchorage, AK, USA, 20-23 September 2009.

17. Wang, L.M.; Shi, Y. Patrol detection for replica attacks on wireless sensor networks. Sensors 2011, 11, 2496-2504.

18. Bonaci, T.; Lee, P.; Bushnell, L.; Poovendran, R. A convex optimization approach for clone detection in wireless sensor networks. Pervasive Mobile Comput. 2012, In press.

19. Jung, J.; Paxon, V.; Berger, A.W.; Balakrishnan, H. Fast Port Detection Using Sequential Hypothesis Testing. In Proceedings of the IEEE Symposium on Security and Privacy, Berkeley, CA, USA, 9-12 May 2004.

20. Xbow Sensor Networks. Available online: http://www.xbow.com/ (accessed on 15 August 2012).

(c) 2012 by the author; licensee MDPI, Basel, Switzerland. This article is an open access article distributed under the terms and conditions of the Creative Commons Attribution license (http://creativecommons.org/licenses/by/3.0/). 